\title{
The malignant impact of socio-economic disparities
}

\author{
Sara Suliman
}

University of Toronto

It is undeniable that the field of oncology has made great strides to optimize methods of early detection and treatment of different malignancies. Recently, technological advancement has even established refined associations between genetic factors such as single nucleotide polymorphisms (SNPs) and proteomic biomarkers, to cancer susceptibility, such as hematologic malignancies ${ }^{[1,2]}$. New personalized treatments to specific oncogenic mutations have also been developed, such as Gleevec; which specifically inhibits overactivated enzymes in chromosomal translocations found in B cell lymphomas ${ }^{[3]}$. Consequently, research in developed countries, such as Canada, has secured greater survival rates due to better detection, heightened awareness of risk factors by the health care community, and more efficacious treatment regimens. On the other hand, little of the newly developed technologies have been efficiently transferred to developing countries in a financially accessible manner to the public ${ }^{[4]}$. In this article, I aim to present a case for the impact of economic inequalities on the ability of patients to access care. The widening economic gap between different classes of society globally has gradually heightened the mortality risk for members of marginalized communities worldwide. Some of the gaps in access to care can be attributed to differing cultural contexts, such as the tendency to self-medicate to avoid visiting doctors ${ }^{[5]}$. However, the care gap remains largely attributable to the cost burden on individuals in most developing countries and in some impoverished areas of developed ones ${ }^{[6]}$.

The common impression that cancer constitutes a relatively minor problem in developing countries relative to infectious pandemics has been steadily shifting in recent years ${ }^{[7]}$. The situation is further exacerbated in developing countries by the relatively poor health infrastructure leading to lower detection, treatment and palliative care. The inevitable consequence is that many patients present with the disease at terminal stages when treatment is more likely futile and costly. In addition, many patients cannot afford the recommended treatments, even if detected early enough to treat. For instance, the scarcity of proficient mammography facilities in Sub-Saharan Africa, and the relatively high cost to the average individual adds to the problem of late diagnosis of breast cancer $^{[8]}$. In central Sudan, the majority of breast cancer patients present at stage III or later with frequent metastasis, rendering medical intervention futile ${ }^{[9]}$. This can also reflect the need to educate the public on the importance of seeking early medical attention, when feasible. For instance, the implementation of cancer advocacy organizations in certain developing countries in Asia, such as Nepal and Pakistan, is expected to increase the rate of early detection and treatment of common neoplasms ${ }^{[10]}$.

The global response to infectious pandemics such as HIV/AIDS in developing countries is gaining momentum and attracting resources, including researchers and heath care professionals globally ${ }^{[11]}$. However, the perception of cancer as a public health emergency in developing countries is still in its infancy. The weak health care infrastructure in developing countries is not coincidental but can partly be traced to global forces centered in the North, like the International Monetary Fund (IMF). The IMF imposed structural adjustment programs on many developing countries in the 1990's, which stipulated curtailment in public spending on social services such as health care as a precondition to receive aid ${ }^{[12,13]}$. The tendency to spend plenty health care resources on infectious diseases such as HIV/AIDS and tuberculosis, although honourable, has certainly eclipsed the need to fight the new cancer epidemic emerging in these countries.

In the short-term, a crucial aspect of cancer treatment in 
poor countries, such as those in Sub-Saharan Africa, is to focus on the treatment of pain and symptoms experienced by terminal cancer patients through a comprehensive palliative care approach, until a sustainable framework of early screening and detection can be funded ${ }^{[14]}$. While the integration of palliative care into national health policy remains a challenge in many developing countries, some positive examples can be learned from some low-resource countries, such as Cuba, where all cancer patients receive primary care from a team of health workers, including physicians, nurses and social workers, at no cost ${ }^{[15]}$.

If the disparities of access to cancer treatments were due to the infrastructure discrepancy between developed and developing countries alone, one would predict that the situation is less gloomy in developed countries. However, in the United States, the absence of fully universal health care system, still render low-income individuals and communities highly susceptible to later detection and poorer prognosis ${ }^{[16]}$. Interestingly, some countries offering universal health care, such as Canada, still exhibit a negative association between socioeconomic status and susceptibility to cancer ${ }^{[17]}$. This phenomenon showcases that basic access to health care, without improving the living standards, is likely insufficient to improve risk and prognosis in impoverished communities.

In summary, socio-economic disparities remain an under-explored influential factor for the prognosis and potential survival of many cancer patients worldwide. As concerned advocates in the cancer research community, we should proceed towards establishing more equitable, and ethical allocation of resources towards the diagnosis and treatment across the board ${ }^{[4]}$. The impressive strides in cancer detection and treatment technologies should not stop. Nonetheless, a new lens addressing the inequities in technology transfer and resource distribution to all citizens of the world needs to be integrated in the cancer research agenda. This will ensure that the goal of eradicating this pandemic is truly genuine and efficacious.

\section{References}

1. Heinrichs, S., C. Li, and A.T. Look, SNP array analysis in hematologic malignancies: avoiding false discoveries. Blood. 115(21): p. 4157-61.

2. Ludwig, J.A. and J.N. Weinstein, Biomarkers in cancer staging, prognosis and treatment selection. Nat Rev Cancer, 2005. 5(11): p. 845-56.
3. Druker, B.J., et al., Activity of a specific inhibitor of the $B C R-A B L$ tyrosine kinase in the blast crisis of chronic myeloid leukemia and acute lymphoblastic leukemia with the Philadelphia chromosome. N Engl J Med, 2001. 344(14): p. 1038-42.

4. Farmer, P., et al., Expansion of cancer care and control in countries of low and middle income: a call to action. Lancet. 376(9747): p. 1186-93.

5. Yousif, M.A., In-home drug storage and utilization habits: a Sudanese study. East Mediterr Health J, 2002. 8(2-3): p. 422-31.

6. Mano, M., The burden of scientific progress: growing inequalities in the delivery of cancer care. Acta Oncol, 2006. 45(1): p. 84-6.

7. Jemal, A., et al., Global cancer statistics. CA Cancer J Clin. 61(2): p. 69-90.

8. Galukande, M. and E. Kiguli-Malwadde, Rethinking breast cancer screening strategies in resource-limited settings. Afr Health Sci. 10(1): p. 89-92.

9. Elgaili, E.M., et al., Breast cancer burden in central Sudan. Int J Womens Health. 2: p. 77-82.

10. Yoo, K.Y., Cancer prevention in the Asia Pacific region. Asian Pac J Cancer Prev. 11(4): p. 839-44.

11. Einterz, R.M., et al., Responding to the HIV pandemic: the power of an academic medical partnership. Acad Med, 2007. 82(8): p. 812-8.

12. Ekwempu, C.C., et al., Structural adjustment and health in Africa. Lancet, 1990. 336(8706): p. 56-7.

13. Sekwat, A., Health financing reform in sub-Saharan Africa: major constraints, goals, and strategies. J Health Care Finance, 2003. 29(3): p. 67-78.

14. Stjernsward, J., Palliative care: the public health strategy. J Public Health Policy, 2007. 28(1): p. 42-55.

15. Sepulveda, C., et al., Palliative Care: the World Health Organization's global perspective. J Pain Symptom Manage, 2002. 24(2): p. 91-6.

16. Ward, E., et al., Cancer disparities by racelethnicity and socioeconomic status. CA Cancer J Clin, 2004. 54(2): p. 78-93.

17. Mackillop, W.J., et al., Associations between community income and cancer incidence in Canada and the United States. Cancer, 2000. 89(4): p. 901-12.

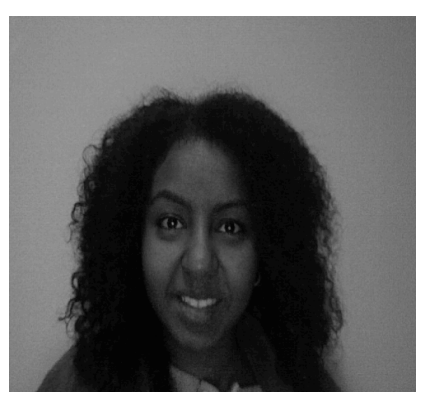

\section{Author Profile}

Sara Suliman is a $\mathrm{PhD}$ Candidate in the Department of Immunology, University of Toronto. She is interested in studying the pathogenesis of childhood leukemias. She is also interested in a number of public health issues, including universal health care provision and primary care in developing countries. 